\title{
Unbiased Minimum-variance Filtering for Input Reconstruction
}

\author{
Harish J. Palanthandalam-Madapusi and Dennis S. Bernstein
}

\begin{abstract}
In this paper, we introduce the concept of input and state observability, that is, conditions under which both the unknown input and state can be estimated from the output measurements. We discuss sufficient and necessary conditions for a discrete-time system to be input and state observable. Next, we derive an unbiased minimum-variance filter to estimate the unknown input and the state, when the state space matrices are known. We show that the Kalman filter and other filters in the literature are special cases of the filter derived in this paper. Finally, we present an illustrative example.
\end{abstract}

\section{INTRODUCTION}

Systems with unknown inputs have received considerable attention in the past [3-8, 10-23, 25-28]. An active area of research is state reconstruction with known model equations and unknown inputs. Among the popular approaches are full-order observers $[4,6,15,16,28]$, reducedorder observers [7, 8, 19,21], geometric approach [3], generalized inverse approach [19,21], trial-and-error approach [26], and the singular value decomposition [8]. A widely used approach is to model the unknown inputs as outputs of a known dynamic system and incorporate the input dynamics with the plant dynamics [1,13]. However, this approach increases the dimension of the observer and is limited to specific types of inputs.

The unknown inputs in a dynamical system may represent unknown external drivers, input uncertainty, state uncertainty, or instrument faults. Thus unknown-input reconstruction has several important applications in uncertainty estimation and fault detection. Input reconstruction also has applications in filtering and coding theory. In some early work, input reconstruction is achieved through system inversion [23, 25]. More recently, methods for input reconstruction using optimal filters are developed in [4, 10, 14, 27].

An unbiased minimum-variance filter for discrete-time stochastic systems with arbitrary unknown inputs is derived in [16]. Finally, [9] presents an alternative derivation of the filter in [16] and uses the filter to reconstruct the unknown inputs. A related filter is presented in [24].

A related problem is the concept of input and state observability, which is the ability to reconstruct the inputs and states using only output measurements. Necessary and sufficient conditions for input and state observability for

This research was supported in part by the National Science Foundation Information Technology Research initiative, through Grant ATM-0325332.

H. J. Palanthandalam-Madapusi and D. S. Bernstein are with the department of Aerospace Engineering at the University of Michigan, Ann Arbor, MI 48109-2140. \{hpalanth,dsbaero\}@umich.edu continuous-time systems in terms of the invariant zeros of the system are presented in $[4,8,12,14,19]$. Input and state observability for discrete-time systems are considered in [14].

In this paper, we adopt a rigorous approach to examine conditions under which both the input and state can be estimated from the output measurements. We discuss necessary and sufficient conditions for a discrete-time system to be input and state observable and derive simple tests for input and state observability. Since no assumptions on the input are made, the unknown input can be either an unmodeled exogenous signal or an unknown function of the states.

Once the necessary and sufficient conditions for input and state observability are presented, we develop optimal filtering techniques that take advantage of input and state observability. The Kalman filter and the filters derived in [16, 24] are shown to be special cases of the unbiased minimumvariance filter derived in this paper.

Finally, we present an example to illustrate the methods.

\section{INPUT AND STATE OBSERVABILITY}

\section{A. No Feedthrough Case}

Consider the time-varying system

$$
\begin{aligned}
x_{k+1} & =A_{k} x_{k}+H_{k} e_{k}, \\
y_{k} & =C_{k} x_{k},
\end{aligned}
$$

where $x_{k} \in \mathbb{R}^{n}, e_{k} \in \mathbb{R}^{p}, y_{k} \in \mathbb{R}^{l}, A_{k} \in \mathbb{R}^{n \times n}, H_{k} \in$ $\mathbb{R}^{n \times p}$, and $C_{k} \in \mathbb{R}^{l \times n}$. Without loss of generality, we assume $l \leq n, \max _{k}\left[\operatorname{rank}\left(C_{k}\right)\right]=l>0$, and $\max _{k}\left[\operatorname{rank}\left(H_{k}\right)\right]=p>0$. No assumption on the inputs $e_{k}$ are made. Hence, the signal $e_{k}$ can either be an exogenous input or a nonlinear function of the states. For the sake of simplicity, we assume that the initial time $k_{0}=0$.

Throughout this paper, $r$ denotes a nonnegative integer. Furthermore, for convenience, every vector or matrix with zero rows or zero columns is an empty matrix. Define the data vectors $y_{r} \in \mathbb{R}^{(r+1) l}$ and $\mathcal{E}_{r} \in \mathbb{R}^{(r+1) p}$ as

$$
y_{r} \triangleq\left[\begin{array}{c}
y_{0} \\
y_{1} \\
\vdots \\
y_{r}
\end{array}\right], \quad \varepsilon_{r} \triangleq\left[\begin{array}{c}
e_{0} \\
e_{1} \\
\vdots \\
e_{r}
\end{array}\right] .
$$

Definition II.1. Let $r \geq 1$. Then the input and state 
unobservable subspace $\mathfrak{U}_{r}$ of (II.1), (II.2) is the subspace

$$
\mathfrak{U}_{r} \triangleq\left\{\left[\begin{array}{c}
x_{0} \\
\mathcal{E}_{r-1}
\end{array}\right] \in \mathbb{R}^{n+r p}: y_{r}=0\right\} .
$$

We define $\Gamma_{r} \in \mathbb{R}^{(r+1) l \times n}, M_{r} \in \mathbb{R}^{(r+1) l \times r p}$, and $\Psi_{r} \in$ $\mathbb{R}^{(r+1) l \times(n+r p)}$ by

$\Gamma_{r} \triangleq\left[\begin{array}{c}C_{0} \\ C_{1} A_{0} \\ C_{2} A_{1} A_{0} \\ \vdots \\ C_{r} A_{r-1} \cdots A_{0}\end{array}\right], \quad M_{r} \triangleq$

$\left[\begin{array}{cccc}0 & 0 & \cdots & 0 \\ C_{1} H_{0} & 0 & \cdots & 0 \\ C_{2} A_{1} H_{0} & C_{2} H_{1} & \cdots & 0 \\ \vdots & \vdots & \ddots & \vdots \\ C_{r} A_{r-1} \cdots A_{1} H_{0} & C_{r} A_{r-1} \cdots A_{2} H_{1} & \cdots & C_{r} H_{r-1}\end{array}\right]$,

and

$$
\Psi_{r} \triangleq\left[\begin{array}{cc}
\Gamma_{r} & M_{r}
\end{array}\right] .
$$

Note that $M_{0}$ is an empty matrix and thus $\Psi_{0}=\Gamma_{0}=C_{0}$. Next, from (II.1), (II.2), we can write

$$
y_{r}=\Gamma_{r} x_{0}+M_{r} \mathcal{E}_{r-1}=\Psi_{r}\left[\begin{array}{c}
x_{0} \\
\varepsilon_{r-1}
\end{array}\right],
$$

so that

$$
\mathfrak{U}_{r}=\mathcal{N}\left(\Psi_{r}\right),
$$

where $\mathcal{N}$ denotes null space. Next, define the positive integer

$$
r_{0} \triangleq \begin{cases}\max \left\{\left\lceil\frac{n-l}{l-p}\right\rceil, 1\right\}, & p<l, \\ 1, & p=l,\end{cases}
$$

where $\lceil a\rceil$ denotes the smallest integer greater than or equal to $a$. Note that $r_{0}$ is not defined in the case $p>l$.

Proposition II.1. Assume that $n \geq 2$ and $p \leq l$. Then $r_{0} \leq n-1$.

Proof. Suppose $p=l$. Then $n-1 \geq 1=r_{0}$. Next, suppose $p<l$. If $\left\lceil\frac{n-l}{l-p}\right\rceil \leq 1$ then $n-1 \geq 1=r_{0}$. If $\left\lceil\frac{n-l}{l-p}\right\rceil>1$, then, since $n-1>n-l$ and $l-p \geq 1$, it follows that $r_{0}=\left\lceil\frac{n-l}{l-p}\right\rceil \leq\lceil n-l\rceil \leq\lceil n-1\rceil=n-1$.

Proposition II.2. Let $r \geq 1$. If $\mathfrak{U}_{r}=\{0\}$, then the following statements hold:

1) $p \leq l$

2) If $p=l$, then $p=l=n$.

3) $r \geq r_{0}$.

4) $\operatorname{rank}\left(\Gamma_{n-1}\right)=n$.

5) $\operatorname{rank}\left(C_{r} H_{r-1}\right)=p$.

Proposition II.3. Assume that either $p<l$ or $p=l=n$. Then $n+r p \leq(r+1) l$ for all $r \geq r_{0}$.
Proof. Suppose $p=l=n$. Then $n+r p=(r+1) l$ for all $r>0$. Next, suppose $p<l$, let $r \geq r_{0}$ and assume $(r+1) l<n+r p$ so that $r l-r p<n-l$. Hence $r<\frac{n-l}{l-p}$, and thus $\left\lceil\frac{n-l}{l-p}\right\rceil \leq r_{0}<\frac{n-l}{l-p}$, which is a contradiction. Thus $n+r p \leq(r+1) l$.

Proposition II.3 implies that if $p<l$ or $p=l=n$, then for all $r \geq r_{0}$ the number of columns of $\Psi_{r}$ is less than or equal to the number of rows of $\Psi_{r}$.

Definition II.2. The system (II.1), (II.2) is input and state observable if $\mathfrak{U}_{r}=\{0\}$ for all $r \geq r_{0}$.

Definition II.2 implies that if (II.1), (II.2) is input and state observable, then, for all $r \geq r_{0}$, the initial condition $x_{0}$ and input sequence $\left\{e_{i}\right\}_{i=0}^{r-1}$ are uniquely determined from the measured output sequence $\left\{y_{i}\right\}_{i=0}^{r}$.

Theorem II.1. The following statements are equivalent:

1) (II.1), (II.2) is input and state observable.

2) For all $r \geq r_{0}, y_{r}=0$ if and only if $\left[\begin{array}{c}x_{0} \\ \varepsilon_{r-1}\end{array}\right]=0$.

3) For all $r \geq r_{0}, \operatorname{rank}\left(\Psi_{r}\right)=n+r p$.

4) $\operatorname{rank}\left(\Psi_{n-1}\right)=n+(n-1) p$ and for all $r \geq r_{0}$, $\operatorname{rank}\left(C_{r} H_{r-1}\right)=p$.

Proof. From Definition II.1 and Definition II.2 it follows that 1$) \Rightarrow 2$ ). Using (II.7), 2) $\Rightarrow 3$ ). To prove 3 ) $\Rightarrow 4$ ), since for all $r \geq r_{0}, \operatorname{rank}\left(\Psi_{r}\right)=n+r p$, it follows that $\operatorname{rank}\left(C_{r} H_{r-1}\right)=p$. Hence, for all $\hat{r} \geq r_{0} \operatorname{rank}\left(\Psi_{\hat{r}}\right)=$ $\operatorname{rank}\left(\Psi_{\hat{r}-1}\right)+p$. Hence, since $n-1 \geq r_{0}$, we have $\operatorname{rank}\left(\Psi_{n-1}\right)=n+(n-1) p$. Finally to show 4$\left.) \Rightarrow 1\right)$, we consider two cases. First, suppose $n=1$. In this case $C_{k}$ and $H_{k}$ are nonzero scalars, and hence it follows that $\operatorname{rank}\left(\Psi_{r}\right)=n+r p$ for all $r \geq r_{0}$ and hence $\mathfrak{U}_{r}=\{0\}$ for all $r \geq r_{0}$. Next, suppose $n \geq 2$. In this case, 4) implies that $\operatorname{rank}\left(\Psi_{r}\right)=\operatorname{rank}\left(\Psi_{r-1}\right)+p$ for all $r \geq r_{0}$. Next, since $n-1 \geq r_{0}$, it follows that, for all $r \geq r_{0}, \operatorname{rank}\left(\Psi_{r}\right)=$ $\operatorname{rank}\left(\Psi_{n-1}\right)+(r-n+1) p$. Thus $\operatorname{rank}\left(\Psi_{r}\right)=n+r p$ for all $r \geq r_{0}$ and hence $\mathfrak{U}_{r}=\{0\}$ for all $r \geq r_{0}$.

Theorem II.1 shows that (II.1), (II.2) is input and state observable if and only if $\Psi_{r}$ has full column rank for all $r \geq r_{0}$. In this case the unique solution of (II.7) is

$$
\left[\begin{array}{c}
x_{0} \\
\mathcal{E}_{r-1}
\end{array}\right]=\Psi_{r}^{\dagger} y_{r}
$$

where $\dagger$ represents the Moore-Penrose generalized inverse $\Psi_{r}^{\dagger}=\left(\Psi_{r}^{\mathrm{T}} \Psi_{r}\right)^{-1} \Psi_{r}^{\mathrm{T}}$.

\section{B. Feedthrough Case}

Next, we consider the system

$$
\begin{aligned}
x_{k+1} & =A_{k} x_{k}+H_{k} e_{k}, \\
y_{k} & =C_{k} x_{k}+G_{k} e_{k},
\end{aligned}
$$

where $G_{k} \in \mathbb{R}^{l \times p}$, while $A_{k}, H_{k}, C_{k}, x_{k}, e_{k}$, and $y_{k}$ are defined as in (II.1), (II.2). Without loss of general- 
ity, we assume $l \leq n, \max _{k}\left[\operatorname{rank}\left(C_{k}\right)\right]=l>0$, and $\max _{k}\left(\operatorname{rank}\left[\begin{array}{c}H_{k} \\ G_{k}\end{array}\right]\right)=p>0$. Due to the term $G_{k} e_{k}$, the output $y_{k}$ is directly affected by $e_{k}$ as well as by the past values of $e_{k}$. Therefore, we have

$$
y_{r}=\bar{\Psi}_{r}\left[\begin{array}{l}
x_{0} \\
\mathcal{E}_{r}
\end{array}\right]
$$

where $\bar{\Psi}_{r} \triangleq\left[\begin{array}{cc}\Gamma_{r} & \bar{M}_{r}\end{array}\right] \in \mathbb{R}^{(r+1) l \times[n+(r+1) p]}$, and $\bar{M}_{r}=$

$\left[\begin{array}{ccccc}G_{0} & 0 & \cdots & 0 & 0 \\ C_{1} H_{0} & G_{1} & \cdots & 0 & 0 \\ \vdots & \vdots & \ddots & \vdots & \\ C_{r-1} A_{r-2} \cdots A_{1} H_{0} & C_{r-1} A_{r-2} \cdots A_{2} H_{1} & \cdots & G_{r-1} & 0 \\ C_{r} A_{r-1} \cdots A_{1} H_{0} & C_{r} A_{r-1} \cdots A_{2} H_{1} & \cdots & C_{r} H_{r-1} & G_{r}\end{array}\right]$.

Furthermore, we have the following definition.

Definition II.3. Let $r \geq 0$. Then the input and state unobservable subspace $\overline{\mathfrak{U}}_{r}$ of (II.11), (II.12) is the subspace

$$
\overline{\mathfrak{U}}_{r} \triangleq\left\{\left[\begin{array}{l}
x_{0} \\
\mathcal{E}_{r}
\end{array}\right] \in \mathbb{R}^{n+(r+1) p}: y_{r}=0\right\} \text {. }
$$

The input and state unobservable subspace is given by $\overline{\mathfrak{U}}_{r}=\mathcal{N}\left(\bar{\Psi}_{r}\right)$. Next, if $p<l$ then define the integer

$$
\bar{r}_{0} \triangleq\left\lceil\frac{n}{l-p}\right\rceil-1 \text {. }
$$

Since $n>l-p$ it follows that $\bar{r}_{0} \geq 1$.

Proposition II.4. Let $r \geq 0$. If $\overline{\mathfrak{U}}_{r}=\{0\}$, then the following statements hold:
1) $p<l$.
2) $n>1$.
3) $r \geq \bar{r}_{0}$.
4) $\operatorname{rank}\left(\Gamma_{n-1}\right)=n$.
5) $\operatorname{rank}\left(G_{r}\right)=p$.

Definition II.4. The system (II.11), (II.12) is input and state observable if $\overline{\mathfrak{U}}_{r}=\{0\}$ for all $r \geq \bar{r}_{0}$.

Theorem II.2. The following statements are equivalent:

1) (II.11), (II.12) is input and state observable.

2) For all $r \geq \bar{r}_{0}, y_{r}=0$ if and only if $\left[\begin{array}{l}x_{0} \\ \mathcal{E}_{r}\end{array}\right]=0$.

3) For all $r \geq \bar{r}_{0} \operatorname{rank}\left(\bar{\Psi}_{r}\right)=n+(r+1) p$.

4) $\operatorname{rank}\left(\bar{\Psi}_{n-1}\right)=n(p+1)$ and for all $k \geq r_{0}$, $\operatorname{rank}\left(G_{k}\right)=p$.

Finally, if (II.11), (II.12) is input and state observable, then Theorem II.2 implies that $\bar{\Psi}_{r}$ is full column rank for all $r \geq \bar{r}_{0}$. In this case the unique solution of (II.13) is

$$
\left[\begin{array}{l}
x_{0} \\
\varepsilon_{r}
\end{array}\right]=\bar{\Psi}_{r}^{\dagger} y_{r}
$$

III. UNBIASED MINIMUM-VARIANCE FILTER FOR INPUT AND STATE OBSERVABLE SyStems

Consider the time-varying system

$$
\begin{aligned}
x_{k+1} & =A_{k} x_{k}+B_{k} u_{k}+H_{k} e_{k}+w_{k}, \\
y_{k} & =C_{k} x_{k}+D_{k} u_{k}+G_{k} e_{k}+v_{k} .
\end{aligned}
$$

where $x_{k}, y_{k}, e_{k}, A_{k}, C_{k}, H_{k}$ and $G_{k}$ are defined as in section 2, while $u_{k} \in \mathbb{R}^{m}, B_{k} \in \mathbb{R}^{n \times m}$ and $D_{k} \in \mathbb{R}^{l \times m}$. We assume that $A_{k}, B_{k}, C_{k}, D_{k}, H_{k}$, and $G_{k}$ are known, while $e_{k}$ is unknown. $w_{k} \in \mathbb{R}^{n}$ and $v_{k} \in \mathbb{R}^{l}$ are unknown Gaussian white noise sequences with known covariances $Q_{k}$ and $R_{k}$ respectively. We say that (III.1), (III.2) is input and state observable if it is input and state observable with $B_{k} \equiv 0$ and $D_{k} \equiv 0$. We assume that (III.1), (III.2) is input and state observable.

We consider a filter of the form

$$
\begin{aligned}
\hat{x}_{k+1 \mid k+1}= & \hat{x}_{k+1 \mid k}+L_{k+1}\left(y_{k+1}-C_{k+1} \hat{x}_{k+1 \mid k}\right. \\
& \left.-D_{k+1} u_{k+1}\right) \\
\hat{x}_{k+1 \mid k}= & A_{k} \hat{x}_{k \mid k}+B_{k} u_{k} .
\end{aligned}
$$

The state estimation error is

$$
\varepsilon_{k} \triangleq x_{k+1}-\hat{x}_{k+1 \mid k+1}
$$

and the error covariance matrix is defined as

$$
P_{k+1 \mid k+1} \triangleq \mathbb{E}\left[\varepsilon_{k+1} \varepsilon_{k+1}^{\mathrm{T}}\right],
$$

where $\mathbb{E}$ is the expected value. The filter is unbiased if and only if

$$
\mathbb{E}\left[x_{k+1}-\hat{x}_{k+1 \mid k+1}\right]=0 .
$$

Furthermore, (III.7) can be written as

$$
\begin{gathered}
\mathbb{E}\left[A_{k} \varepsilon_{k}+H_{k} e_{k}+w_{k}-L_{k+1}\left(C_{k+1} A_{k} \varepsilon_{k}\right.\right. \\
+C_{k+1} H_{k} e_{k}+C_{k+1} w_{k} \\
\left.\left.+v_{k+1}+G_{k+1} e_{k+1}\right)\right]=0 .
\end{gathered}
$$

Since $e_{k}$ is arbitrary, (III.8) implies

$$
L_{k+1} G_{k+1}=0,
$$

and

$$
\left(I-L_{k+1} C_{k+1}\right) H_{k}=0 .
$$

Lemma III.1. If (III.9) and (III.10) are satisfied, the error covariance matrix $P_{k+1 \mid k+1}$ is given by

$$
\begin{aligned}
P_{k+1 \mid k+1}= & L_{k+1} \tilde{R}_{k+1} L_{k+1}^{\mathrm{T}}-F_{k+1} L_{k+1}^{\mathrm{T}} \\
& -L_{k+1} F_{k+1}^{\mathrm{T}}+P_{k+1 \mid k}
\end{aligned}
$$

where

$$
\begin{aligned}
P_{k+1 \mid k} & \triangleq A_{k} P_{k \mid k} A_{k}^{\mathrm{T}}+Q_{k} \\
\tilde{R}_{k+1} & \triangleq C_{k+1} P_{k+1 \mid k} C_{k+1}^{\mathrm{T}}+R_{k+1} \\
F_{k+1} & \triangleq P_{k+1 \mid k} C_{k+1}^{\mathrm{T}} .
\end{aligned}
$$




\section{Proof.}

$$
\begin{aligned}
P_{k+1 \mid k+1} & \\
= & \mathbb{E}\left[\varepsilon_{k+1} \varepsilon_{k+1}^{\mathrm{T}}\right] \\
= & \mathbb{E}\left[\left(A_{k} x_{k}+H_{k} e_{k}+w_{k}-A_{k} \hat{x}_{k \mid k}\right.\right. \\
& -L_{k+1}\left(C_{k+1} A_{k} x_{k}+C_{k+1} H_{k} e_{k}+C_{k+1} w_{k}\right. \\
& \left.\left.+G_{k} e_{k+1}+v_{k+1}-C_{k+1} A_{k} \hat{x}_{k \mid k}\right)\right) \\
& \times\left(A_{k} x_{k}+H_{k} e_{k}+w_{k}-A_{k} \hat{x}_{k \mid k}\right. \\
& -L_{k+1}\left(C_{k+1} A_{k} x_{k}+C_{k+1} H_{k} e_{k}+C_{k+1} w_{k}\right. \\
& \left.\left.\left.+G_{k} e_{k+1}+v_{k+1}-C_{k+1} A_{k} \hat{x}_{k \mid k}\right)\right)^{\mathrm{T}}\right] . \quad \text { III }
\end{aligned}
$$

Since (III.9) and (III.10) are satisfied, (III.15) becomes

$$
\begin{aligned}
P_{k+1 \mid k+1} & \\
= & \mathbb{E}\left[\left(\left[I-L_{k+1} C_{k+1}\right] A_{k} \varepsilon_{k}+L_{k+1} v_{k+1}\right.\right. \\
& \left.-\left[I-L_{k+1} C_{k+1}\right] w_{k}\right) \\
& \times\left(\left[I-L_{k+1} C_{k+1}\right] A_{k} \varepsilon_{k}+L_{k+1} v_{k+1}\right. \\
& \left.\left.-\left[I-L_{k+1} C_{k+1}\right] w_{k}\right)^{\mathrm{T}}\right] .
\end{aligned}
$$

Noting that $\mathcal{E}\left[\varepsilon_{k} w_{k}^{\mathrm{T}}\right]=0, \mathbb{E}\left[\varepsilon_{k} v_{k+1}^{\mathrm{T}}\right]=0$ and $\mathbb{E}\left[w_{k} v_{k+1}^{\mathrm{T}}\right]=$ 0 , we have that

$$
\begin{aligned}
& P_{k+1 \mid k+1}=L_{k+1} R_{k+1} L_{k+1}^{\mathrm{T}} \\
& \quad+\left(I-L_{k+1} C_{k+1}\right)\left[A_{k} P_{k \mid k} A_{k}^{\mathrm{T}}+Q_{k}\right] \\
& \quad \times\left(I-L_{k+1} C_{k+1}\right)^{\mathrm{T}}
\end{aligned}
$$

Furthermore, using (III.12) - (III.14) and (III.17), it follows that (III.11) holds.

Next, we define the cost function $J$ as the trace of the error covariance matrix

$$
J\left(L_{k+1}\right)=\operatorname{tr} \mathbb{E}\left[\varepsilon_{k+1} \varepsilon_{k+1}^{\mathrm{T}}\right]=\operatorname{tr} P_{k+1 \mid k+1} .
$$

Theorem III.1. The unbiased minimum-variance gain $L_{k+1}$ in the filter (III.3) that minimizes the cost function (III.18) subject to the constraints (III.9) and (III.10) is given by

$$
\begin{aligned}
& L_{k+1}=\left(F_{k+1}\right. \\
& \left.\quad-\Omega_{k+1}\left(\Phi_{k+1}^{\mathrm{T}} \tilde{R}_{k+1}^{-1} \Phi_{k+1}^{\mathrm{T}}\right)^{-1} \Phi_{k+1}^{\mathrm{T}}\right) \tilde{R}_{k+1}^{-1},
\end{aligned}
$$

where

$$
\begin{aligned}
\Phi_{k+1} & \triangleq\left[\begin{array}{ll}
-G_{k+1} & V_{k+1}
\end{array}\right] \\
V_{k+1} & \triangleq C_{k+1} H_{k}, \\
\Omega_{k+1} & \triangleq\left[\begin{array}{ll}
0_{n \times p} & H_{k}
\end{array}\right]-F_{k+1} \tilde{R}_{k+1}^{-1} \Phi_{k+1}
\end{aligned}
$$

Proof. The cost function $J$ can be written as

$$
\begin{aligned}
J\left(L_{k+1}\right)= & \operatorname{tr} P_{k+1 \mid k+1} \\
= & \operatorname{tr}\left[L_{k+1} \tilde{R}_{k+1} L_{k+1}^{\mathrm{T}}-F_{k+1} L_{k+1}^{\mathrm{T}}\right. \\
& \left.-L_{k+1} C_{k+1} P_{k+1 \mid k}+P_{k+1 \mid k}\right] .
\end{aligned}
$$

Thus the optimization problem is to minimize the cost function (III.23) subject to the constraints (III.9) and (III.10). If $\Lambda_{k} \in \mathbb{R}^{n \times 2 q}$ is the matrix of Lagrange multipliers, then the Lagrangian is

$$
\begin{aligned}
& \mathcal{L}\left(L_{k+1}\right) \triangleq J\left(L_{k+1}\right) \\
& \quad+2 \operatorname{tr}\left(\left[L_{k+1} G_{k+1} \quad\left(I-L_{k+1} C_{k+1}\right) H_{k}\right] \Lambda_{k+1}^{\mathrm{T}}\right) .
\end{aligned}
$$

Differentiating with respect to $L_{k+1}$ and setting it to zero, we get

$$
\begin{aligned}
& 2 \tilde{R}_{k+1} L_{k+1}^{\mathrm{T}}-2 C_{k+1} P_{k+1 \mid k} \\
& \quad+2\left[\begin{array}{ll}
G_{k+1} & -C_{k+1} H_{k}
\end{array}\right] \Lambda_{k+1}^{\mathrm{T}}=0
\end{aligned}
$$

while differentiating with respect to $\Lambda_{k+1}$ and setting it to zero yields the constraints (III.9) and (III.10). Further, assuming $R_{k}$ to be positive definite, we write (III.25) as

$$
L_{k+1}^{\mathrm{T}}=\tilde{R}_{k+1}^{-1}\left(C_{k+1} P_{k+1 \mid k}-\Phi_{k+1} \Lambda_{k+1}^{\mathrm{T}}\right) .
$$

Using (III.26) in (III.9) and (III.10), we obtain the matrix of Lagrange multipliers

$$
\Lambda_{k+1}=\Omega_{k+1}\left(\Phi_{k+1}^{\mathrm{T}} \tilde{R}_{k+1}^{-1} \Phi_{k+1}^{\mathrm{T}}\right)^{-1} .
$$

Substituting (III.27) into (III.26), yields the optimal solution for $L_{k+1}$ as

$$
\begin{aligned}
L_{k+1} & =\left(F_{k+1}\right. \\
& \left.-\Omega_{k+1}\left(\Phi_{k+1}^{\mathrm{T}} \tilde{R}_{k+1}^{-1} \Phi_{k+1}^{\mathrm{T}}\right)^{-1} \Phi_{k+1}^{\mathrm{T}}\right) \tilde{R}_{k+1}^{-1} .
\end{aligned}
$$

It is straightforward to check that $L_{k+1}$ given by (III.19) satisfies the constraints (III.9) and (III.10). We now show that the filters presented in $[16,24]$ and the Kalman filter are special cases of the filter (III.19).

Proposition III.1. Suppose $H_{k} \equiv 0$ in (III.1), then the unbiased minimum-variance filter gain $L_{k+1}$ satisfying (III.9) is given by

$$
\begin{gathered}
L_{k+1}=\left[F_{k+1}-F_{k+1} \tilde{R}_{k+1}^{-1} G_{k+1}\right. \\
\left.\times\left(G_{k+1}^{\mathrm{T}} \tilde{R}_{k+1}^{-1} G_{k+1}\right)^{-1} G_{k+1}^{\mathrm{T}}\right] \tilde{R}_{k+1}^{-1} .
\end{gathered}
$$

Furthermore, the covariance update equation is given by

$$
\begin{gathered}
P_{k+1 \mid k+1}=P_{k+1 \mid k}-F_{k+1} \tilde{R}_{k+1}^{-1}\left[I-G_{k+1}\right. \\
\left.\left(G_{k+1}^{\mathrm{T}} \tilde{R}_{k+1}^{-1} G_{k+1}\right)^{-1} G_{k+1}^{\mathrm{T}} \tilde{R}_{k+1}^{-1}\right] F_{k+1}^{\mathrm{T}}
\end{gathered}
$$

Proof. Substituting $H_{k}=0$ in equation (III.19) yields (III.28). Next, we note that when $H_{k}=0$ and (III.9) is statisfied, $P_{k+1 \mid k+1}$ is again given by (III.11). Furthermore, using the expression for $L_{k+1}$ from (III.28) in (III.11), we get (III.29).

Proposition III.1 concides with the output-correction filter developed in [24]. Next, the following result is given in [16]. 
Proposition III.2. Suppose $G_{k} \equiv 0$ in (III.2) and assume (III.1), (III.2) is input and state observable. Then the unbiased minimum-variance filter gain $L_{k+1}$ satisfying (III.10) is

$$
L_{k+1}=H_{k} \Pi_{k}+F_{k+1} \tilde{R}_{k+1}^{-1}\left(I-V_{k+1} \Pi_{k}\right),
$$

where $\Pi_{k}$ is defined as

$$
\Pi_{k} \triangleq\left(V_{k+1}^{\mathrm{T}} \tilde{R}_{k+1}^{-1} V_{k+1}\right)^{-1} V_{k+1}^{\mathrm{T}} \tilde{R}_{k+1}^{-1} .
$$

Furthermore, the covariance update equation becomes

$$
\begin{aligned}
& P_{k+1 \mid k+1}=P_{k+1 \mid k}-F_{k+1} \tilde{R}_{k+1}^{-1} F_{k+1}^{\mathrm{T}}+ \\
& \quad\left(H_{k}-F_{k+1} \tilde{R}_{k+1}^{-1} V_{k+1}\right)\left(V_{k+1}^{\mathrm{T}} \tilde{R}_{k+1}^{-1} V_{k+1}\right)^{-1} \\
& \quad \times\left(H_{k}-F_{k+1} \tilde{R}_{k+1}^{-1} V_{k+1}\right)^{\mathrm{T}} .
\end{aligned}
$$

Proof. The proof follows directly from Proposition III.1 and (III.11).

Proposition III.3. If $G_{k}=0$ and $H_{k}=0$ in (III.1), (III.2), then the unbiased minimum-variance filter gain $L_{k+1}$ reduces to the Kalman filter gain

$$
L_{k+1}=F_{k+1} \tilde{R}_{k+1}^{-1},
$$

and the covariance update equation reduces to the Kalman filter covariance update equation

$$
P_{k+1 \mid k+1}=P_{k+1 \mid k}-F_{k+1} \tilde{R}_{k+1}^{-1} F_{k+1}^{\mathrm{T}} .
$$

Proof. Setting $H_{k}=0$ and $G_{k}=0$ in (III.19) and (III.11), we get (III.33) and (III.34) respectively.

So far, we have discussed unbiased estimation of the state $x_{k}$ in the presence of arbitrary unknown inputs $e_{k}$. Next, we discuss how the unknown inputs $e_{k}$ are estimated, using the unbiased estimates $\hat{x}_{k \mid k}$ of the states $x_{k}$.

Proposition III.4. Consider (III.1), (III.2), and suppose that $\hat{x}_{k \mid k}$ is an unbiased estimate of $x_{k}$ in (III.1). Then

$$
\hat{e}_{k}=G_{k}^{\dagger}\left(y_{k}-C_{k} \hat{x}_{k \mid k}-D_{k} u_{k}\right),
$$

is an unbiased estimate of $e_{k}$.

Proof. Since $l>p$, we can define $\hat{e}_{k}$ as

$$
\hat{e}_{k}=G_{k}^{\dagger}\left(y_{k}-C_{k} \hat{x}_{k \mid k}-D_{k} u_{k}\right),
$$

Taking expected value on both sides of (III.36) yields

$$
\mathbb{E}\left[\hat{e}_{k}\right]=\mathbb{E}\left[G_{k}^{\dagger}\left(G_{k} e_{k}+C_{k} \varepsilon_{k}+v_{k}\right)\right]
$$

Since $\hat{x}_{k \mid k}$ is an unbiased estimate of $x_{k}$, the state estimation error $\varepsilon_{k}$ satisfies

$$
\mathbb{E}\left[\varepsilon_{k}\right]=0 .
$$

Using (III.38) and noting that $v_{k}$ is zero mean, (III.37) becomes

$$
\begin{aligned}
\mathbb{E}\left[\hat{e}_{k}\right] & =\mathbb{E}\left[G_{k}^{\dagger} G_{k} e_{k}\right] \\
& =\mathbb{E}\left[e_{k}\right] .
\end{aligned}
$$

Proposition III.5. Consider (III.1), (III.2) and let $G_{k}=$ 0 . Suppose that $\hat{x}_{k \mid k}$ is an unbiased estimate of the states $x_{k}$ of (III.1). Then

$$
\hat{e}_{k}=H_{k}^{\dagger} L_{k+1}\left(y_{k+1}-C_{k+1} \hat{x}_{k+1 \mid k}-D_{k+1} u_{k+1}\right),
$$

is an unbiased estimate of $e_{k}$.

Proof. Since $l \geq p$, we can define $\hat{e}_{k}$ as

$$
\hat{e}_{k}=H_{k}^{\dagger} L_{k+1}\left(y_{k+1}-C_{k+1} \hat{x}_{k+1 \mid k}-D_{k+1} u_{k+1}\right) \text {, }
$$

where $\dagger$ denotes the Moore-Penrose generalized inverse. Next, we use (III.3) and (III.40) to get

$$
\begin{aligned}
\hat{e}_{k} & =H_{k}^{\dagger}\left(\hat{x}_{k+1 \mid k+1}-\hat{x}_{k+1 \mid k}\right) \\
& =H_{k}^{\dagger}\left(x_{k+1}+\varepsilon_{k+1}-A_{k} \hat{x}_{k \mid k}-B_{k} u_{k}\right) \\
& =H_{k}^{\dagger}\left(x_{k+1}-A_{k} x_{k}-B_{k} u_{k}+\varepsilon_{k+1}-A_{k} \varepsilon_{k}\right) \\
& =H_{k}^{\dagger}\left(H_{k} e_{k}+w_{k}+\varepsilon_{k+1}-A_{k} \varepsilon_{k}\right) .
\end{aligned}
$$

Further, taking expected value on both sides of (III.41), yields

$$
\mathbb{E}\left[\hat{e}_{k}\right]=\mathbb{E}\left[H_{k}^{\dagger}\left(H_{k} e_{k}+w_{k}+\varepsilon_{k+1}-A_{k} \varepsilon_{k}\right)\right],
$$

Finally, noting that $\mathbb{E}\left[\varepsilon_{k}\right]=0$ and the fact that $w_{k}$ is zeromean, we get

$$
\mathbb{E}\left[\hat{e}_{k}\right]=H_{k}^{\dagger} H_{k} \mathbb{E}\left[e_{k}\right]=\mathbb{E}\left[e_{k}\right]
$$

\section{EXAMPLE}

We consider a discrete-time model of the Van der Pol oscillator

$$
\left[\begin{array}{l}
x_{1, k+1} \\
x_{2, k+1}
\end{array}\right]=\left[\begin{array}{c}
x_{1, k}+T_{s} x_{2, k} \\
x_{2, k}+T_{s}\left[\left(1-x_{1, k}^{2}\right) x_{2, k}-x_{1, k}\right]
\end{array}\right],
$$

where $T_{s}$ is the sampling interval. We assume that the linear part of the dynamics is known perfectly, that is, the initial model is the linear part of the equations while $T_{s} x_{1, k}^{2} x_{2, k}$ is assumed to be unknown and thus acts as the unknown input $e_{k}$. Measurements of the state $x_{2}$ are available, thus the output matrix is $C_{k}=\left[\begin{array}{ll}0 & 1\end{array}\right]$. Since the nonlinear term appears only in the equation of the second state we take $H_{k}=\left[\begin{array}{l}0 \\ 1\end{array}\right]$. Figure 1 shows a plot of the actual states, the states from an open-loop simulation of the known model, and the estimates of the states from the unbiased minimum-variance filter. It is seen from the plot that the state estimates from the unbiased minimum-variance filter based on the known model approximates the actual states closely. Once the estimates of the states are obtained we then obtain a 
least squares estimate $\hat{e}_{k}$ of the unknown signal $e_{k}$ by using (III.39). Figure 2 shows the actual unknown signal $e_{k}$ and the estimate $\hat{e}_{k}$ of the unknown signal.

\section{COnClusions}

In this paper, we introduced the concept of input and state observability, that is, conditions under which both the unknown input and state can be estimated from the output measurements. We discussed sufficient and necessary conditions for a discrete-time system to be input and state observable. Next, we derived an unbiased minimum-variance filter to estimate the unknown input and the state. Finally, we presented an illustrative example.

\section{REFERENCES}

[1] B. W. Bequette. Optimal estimation of blood glucose. In Proc. of the IEEE 30th Annual Northeast Bioengineering Conf., pages 77-78, 2004.

[2] D. S. Bernstein. Matrix Mathematics: Theory, Facts, and Formulas with Application to Linear Systems Theory. Princeton University Press, 2005.

[3] S. P. Bhattacharyya. Observer design for linear systems with unknown inputs. IEEE Trans. on Automatic Contr., AC-23:483-484, 1978.

[4] M. Corless and J. Tu. State and input estimation for a class of uncertain systems. Automatica, 34(6):757-764, 1998.

[5] M. Darouach. On the novel approach to the design of unknown input observers. IEEE Trans. on Automatic Contr., 39(3):698-699, March 1994.

[6] M. Darouach, M. Zasadzinski, and S. J. Xu. Full-order observers for linear systems with unknown inputs. IEEE Trans. on Automatic Contr., 39(3):606-609, March 1994.

[7] H. E. Emara-Shabaik. Filtering of linear systems with unknown inputs. Trans. of the ASME, J. of Dyn. Sys., Meas., and Contr., 125:482-485, September 2003.

[8] F. W. Fairman, S. S. Mahil, and L. Luk. Disturbance decoupled observer design via singular value decomposition. IEEE Trans. on Automatic Contr., AC-29(1):84-86, January 1984.

[9] S. Gillijns and B. De Moor. Unbiased minimum-variance input and state estimation for linear discrete-time stochastic systems. Internal Report ESAT-SISTA/TR 05-228, Katholieke Universiteit Leuven, Leuven, Belgium, November 2005.

[10] J. D. Glover. The linear estimation of completely unknown systems. IEEE Trans. on Automatic Contr., pages 766-767, December 1969.

[11] Y. Guan and M. Saif. A novel approach to the design of unknown input observers. IEEE Trans. on Automatic Contr., 36(5):632-635, May 1991.

[12] M. L. J. Hautus. Strong detectability and observers. Lin. Algebra and its Appl., 50:353-368, 1983.

[13] G. Hostetter and J. S. Meditch. Observing systems with unmeasurable inputs. IEEE Trans. on Automatic Contr., AC-18:307-308, June 1973.

[14] M. Hou and R. J. Patton. Input observability and input reconstruction. Automatica, 34(6):789-794, 1998.

[15] M. Hou and R. J. Patton. Optimal filtering for systems with unknown inputs. IEEE Trans. on Automatic Contr., 43(3):445-449, March 1998.

[16] P. K. Kitanidis. Unbiased Minimum-variance Linear State Estimation. Automatica, 23(6):775-578, 1987.

[17] N. Kobayashi and T. Nakamizo. An observer design for linear systems with unknown inputs. Int. J. Contr., 35(4):605-619, 1982.

[18] I. Kolmanovsky, I. Sivergina, and J. Sun. Simultaneous Input and Parameter Estimation With Input Observers and Set-membership Parameter Bounding: Theory and an Automotive Application. Int. J. Adaptive Contr. and Signal Processing, 2006.

[19] P. Kudva, N. Viswanadham, and A. Ramakrishna. Observers for linear systems with unknown inputs. IEEE Trans. on Automatic Contr., 25(1):113-115, February 1980.

[20] J. E. Kurek. The state vector reconstruction for linear systems with unknown inputs. IEEE Trans. on Automatic Contr., AC-28(12):11201122, December 1983.
[21] R. J. Miller and R. Mukundan. On designing reduced-order observers for linear time-invariant systems subject to unknown inputs. Int. J. Contr., 35(1):183-188, January 1982.

[22] B. P. Molinari. A strong controllability and observability in linear multivariable control. IEEE Trans. on Automatic Contr., pages 761764, October 1976.

[23] P. J. Moylan. Stable inversion of linear systems. IEEE Trans. on Automatic Contr., pages 74-78, February 1977.

[24] H. Palanthandalam-Madapusi, S. Gillijns, B. De Moore, and D. S. Bernstein. System identification for nonlinear model updating. In Proc. of Amer. Contr. Conf., Minneapolis, MN, June 2006.

[25] M. K. Sain and J. L. Massey. Invertibility of linear time-invariant dynamical systems. IEEE Trans. on Automatic Contr. AC-14(2):141149, April 1969.

[26] S. H. Wang, E. J. Davison, and P. Dovato. Observing the states of systems with unmeasurable disturbances. IEEE Trans. on Automatic Contr., AC-20:716-717, 1975.

[27] Y. Xiong and M. Saif. Unknown disturbance inputs estimation based on a state functional observer design. Automatica, 39:1389-1398, 2003.

[28] F. Yang and R. W. Wilde. Observers for linear systems with unknown inputs. IEEE Trans. on Automatic Contr., 33(7):677-681, July 1988.
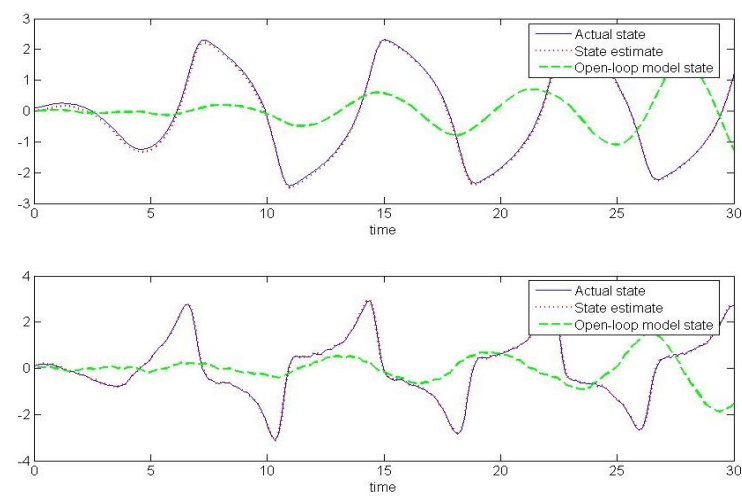

Fig. 1. Discrete-time Van-der Pol Oscillator Example. Comparison of the actual states of the system, the state estimates from the unbiased minimum-variance filter, and the states from an openloop simulation of the known model equations.

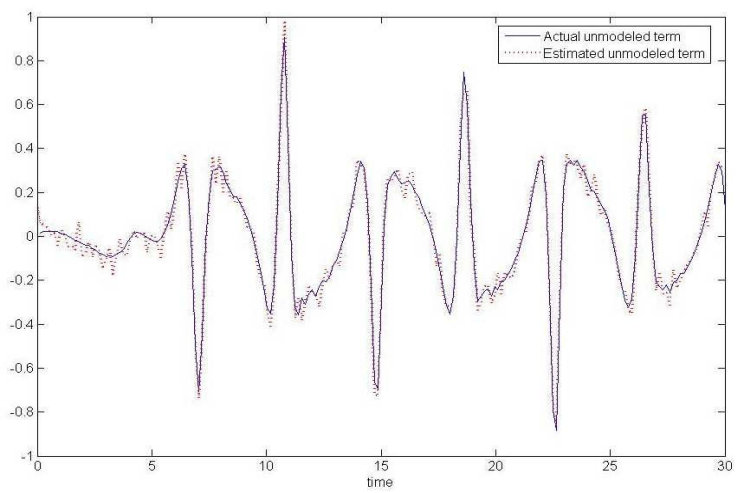

Fig. 2. Discrete-time Van-der Pol Oscillator Example. A plot showing the actual unknown input and the estimated unknown input. 